\title{
The resection angle in apical surgery: a CBCT assessment
}

\author{
Thomas von Arx ${ }^{1}$ - Simone F. M. Janner ${ }^{1}$ - Simon S. Jensen ${ }^{1,2} \cdot$ Michael M. Bornstein ${ }^{1}$
}

Received: 11 May 2015 / Accepted: 14 December 2015 / Published online: 22 December 2015

(C) Springer-Verlag Berlin Heidelberg 2015

\begin{abstract}
Objectives The primary objective of the present radiographic study was to analyse the resection angle in apical surgery and its correlation with treatment outcome, type of treated tooth, surgical depth and level of root-end filling.

Materials and methods In the context of a prospective clinical study, cone beam computed tomography (CBCT) scans were taken before and 1 year after apical surgery to measure the angle of the resection plane relative to the longitudinal axis of the root. Further, the surgical depth (distance from the buccal cortex to the most lingual/palatal point of the resection plane) as well as the level of the root-end filling relative to the most coronal point of the cut root face was determined. Treated teeth were categorized into four groups (maxillary and mandibular anterior and posterior teeth). The final material comprised 62 treated roots in 55 teeth.

Results The mean calculated resection angle of all roots was $17.7^{\circ} \pm 11.4^{\circ}$ (range $-9.6^{\circ}$ to $43.4^{\circ}$ ). Anterior maxillary roots presented the highest mean angle $\left(25.8^{\circ} \pm 10.3^{\circ}\right)$ that was significantly different from the mean angle in posterior maxillary roots $\left(10.7^{\circ} \pm 9.4^{\circ} ; p<0.001\right)$ and from the mean angle in posterior mandibular roots $\left(15.1 \pm 9.8^{\circ} ; p<0.05\right)$. In roots with a shallow resection angle $\left(\leq 20^{\circ}\right)$, the rate of healed cases was higher than in roots with an acute resection angle $\left(>20^{\circ}\right)$, however without reaching statistical significance $(p=0.0905)$.
\end{abstract}

Thomas von Arx

thomas.vonarx@zmk.unibe.ch

1 Department of Oral Surgery and Stomatology, School of Dental Medicine, University of Bern, Freiburgstrasse 7,

CH-3010 Bern, Switzerland

2 Department of Oral and Maxillofacial Surgery, Copenhagen University Hospital, Copenhagen, Denmark
Angles did not correlate either with the surgical depth or with the retrofilling length.

Conclusions Statistically significant differences were observed comparing resection angles of different tooth groups. However, the angle had no significant effect on treatment outcome.

Clinical relevance Contrary to common belief, the resection angle in maxillary anterior teeth was greater than in the other teeth. The surgeon is advised to pay attention to the resection angle when bevelling maxillary anterior teeth in apical surgery.

Keywords Apical surgery $\cdot$ Root-end resection $\cdot$ Resection angle $\cdot$ Cone beam computed tomography

\section{Introduction}

Modern apical surgery includes the use of microsurgical techniques utilizing a surgical microscope and microtips for rootend cavity preparation. Compared to the traditional technique without visual enhancement and use of rotary instruments for root-end cavity preparation, modern apical surgery allows a reduction of the size of the bony access window and a resection angle ideally perpendicular to the long axis of the root [1]. The main objectives of root-end resection are to remove the apical delta of the root canal system and to create a cut root face for inspection and root-end management. However, the apical root portion often shows marked variations in structure and may as such represent special challenges during apical surgery [2]. It has been experimentally shown that steep bevelling (acute resection angle) will not completely remove the apical ramifications [1]. Furthermore, the area of the cut root face and the number of patent dentinal tubules are greater in an acute compared to a shallow resection angle [3]. As a 
consequence, leakage of bacteria and toxins from the root canal system may persist in situations with an acute bevel and/or an insufficient length of the root-end filling [4].

Traditionally, radiographic examination of apical surgery at follow-up appointments is performed with periapical radiography (PA). The two-dimensional imaging modality, however, does not allow the assessment of the resection angle and its possible influence on the clinical outcome. Further, PA may overestimate the length of the root-end filling due to radiographic angulation and image distortion [4]. Recently, threedimensional radiography, i.e. cone beam computed tomography $(\mathrm{CBCT})$ has been utilized to assess the "radiographic healing" following apical surgery $[5,6]$. CBCT also allows assessment of the periapical healing in the bucco-lingual plane, supplementing the typical healing analysis in the mesio-distal plane of PA.

The primary objective of the present radiographic study was to evaluate the resection angle of the cut root face in apical surgery using postoperative CBCT scans and its effect on treatment outcome. Secondary outcome measures included the surgical depth, i.e. the distance along the cut root face from the buccal bone surface to the lingual root outline, as well as the length of the root-end filling.

\section{Material and methods}

Patients with first-time apical surgery were enrolled in this prospective study that had been approved by the Institutional Review Board (Ethic Committee of the State of Bern/Switzerland, approval number KEK-BE 098/11) [7]. All patients had been referred by general practitioners or by institutional staff. They were informed about the treatment alternatives, surgical procedure and follow-up appointments. Further, patients were notified about the pre- and postsurgical radiographic examinations including periapical radiography and $\mathrm{CBCT}$ imaging. CBCT was taken according to the study protocol, independently of the current guidelines for preoperative diagnostics in apical surgery. Patients signed informed consent papers according to the Declaration of Helsinki.

Apical surgery was performed in concurrence with the microsurgical approach, i.e. using a surgical microscope as well as microinstruments for root-end cavity preparation. Details of the surgical technique have been presented previously [8]. Osteotomies for access windows were made with round burs. Root-end resections were performed at $3 \mathrm{~mm}$ from the root apex either by cutting down the root tip with round burs or by separating the root tip with a fissure bur. In cases with long metallic posts or screws, the resection level was placed closer to the apex in order to allow for sufficient depth of root-end preparation and filling. The actual bevelling of the resection plane was carried out with a cylindrical drill (diameter $1.2 \mathrm{~mm}$, working length $4.2 \mathrm{~mm}$; Komet, Besigheim,

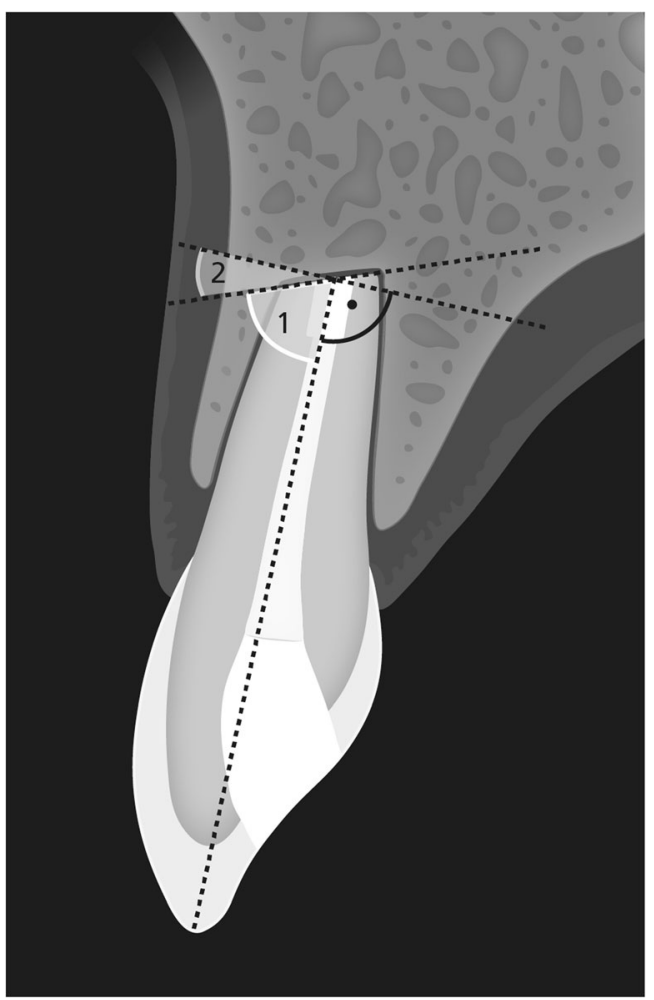

Fig. 1 Schematic illustration of the resection angle in the bucco-lingual plane $($ angle $1=$ angle measured with software tool; angle $2=$ calculated angle, i.e. $90^{\circ}$ minus angle 1 )

Germany) having flutes oriented parallel to the long axis of the bur. Following haemostasis, root-end cavities were prepared along the longitudinal axis of the root using ultrasonic-driven microtips (Endo Success, Satelec, Acteon Group, Merignac, France). Retropreparations were filled with mineral trioxide aggregate (ProRoot MTA, Dentsply Tulsa Dental, Tulsa, OK, USA). A rigid endoscope was routinely used intraoperatively for inspection of the cut root face, retropreparation and retrofilling.

CBCT imaging was performed before and 1 year after apical surgery. The CBCT images were obtained with the 3D Accuitomo 170 (J. Morita Corporation, Kyoto, Japan). The size of the field of view (FOV) was $4 \times 4 \mathrm{~cm}$ or $6 \times 6 \mathrm{~cm}$ for preoperative and $4 \times 4 \mathrm{~cm}$ for postoperative $\mathrm{CBCT}$, respectively. Thus, a small field of view was chosen for all CBCT scans performed in this study [9]. The parameters of the recordings were $3.0 \mathrm{~mA}$ and $80 \mathrm{kV}$ with an exposure time of $17.5 \mathrm{~s}$. CBCT images were evaluated on a Dell 380 workstation (Dell SA, Geneva, Switzerland) and a 19-in. Eizo monitor with a resolution of $1280 \times 1024$ pixels (Eizo Nanao AD, Wädenswil, Switzerland).

The CBCT images were evaluated by one experienced clinician (TvA). Reformatting of the CBCT scans included first aligning the "bucco-lingual reference line" in the axial plane through the centre of the root, then adjusting the "apico-coronal reference line" in the mesio-distal view (coronal plane for 
anterior and sagittal plane for posterior teeth, respectively) through the centre of the root, and finally evaluating the resulting bucco-lingual view. All measurements were taken twice (at least 3 weeks apart) to assess the intra-observer reliability. The means of the two measurements were used for further analysis. The following parameters were determined using the 1-year CBCT scans and measurement tools of the software (i-Dixel version 1.8, Morita, Kyoto, Japan):

- $\quad$ The resection angle $\left({ }^{\circ}\right)$ (Fig. 1): The angle was measured between the long axis of the root and the resection plane; this value was subtracted from $90^{\circ}$ to calculate the resection angle relative to a reference line perpendicular to the long axis of the root.

- The surgical depth (mm) (Fig. 2): The distance from the buccal surface of the bone cortex to the most lingual point of the resection plane along the cut root face (in cases with missing buccal bone at the resection level, the surgical depth was identical with the length of the cut root face in the bucco-lingual direction).

- The length of the root-end filling (Fig. 3): The shortest (most apical) level of the retrofilling relative to a reference line perpendicular to the long axis of the root and through the most "coronal" point of the resection plane. The length of the retrofilling was categorized according to

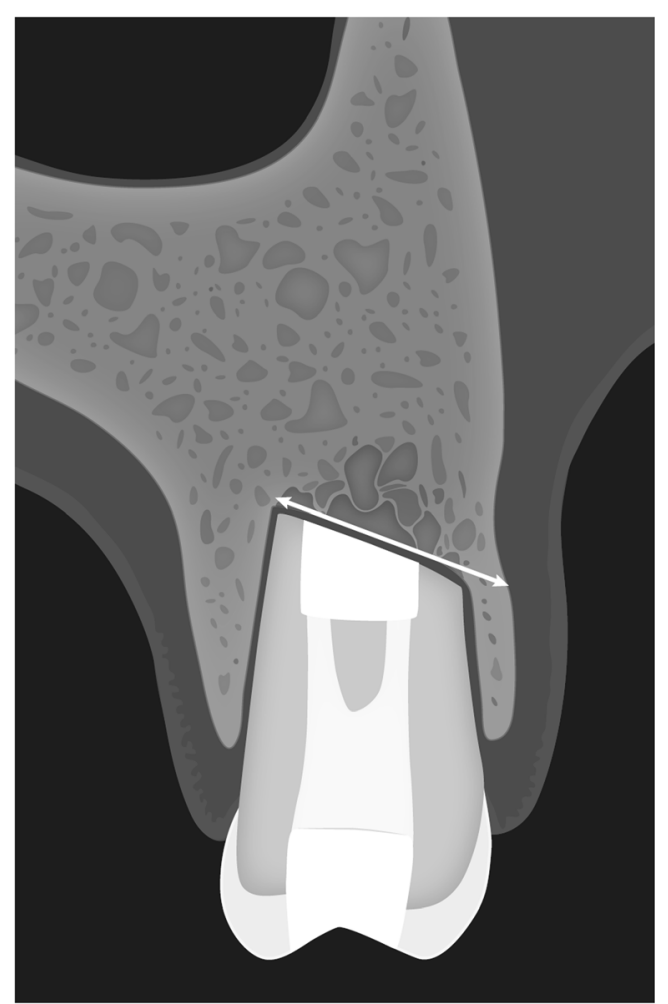

Fig. 2 Schematic illustration of "surgical depth" (double white arrow), the distance from the buccal bone surface to the most lingual point of the resection plane

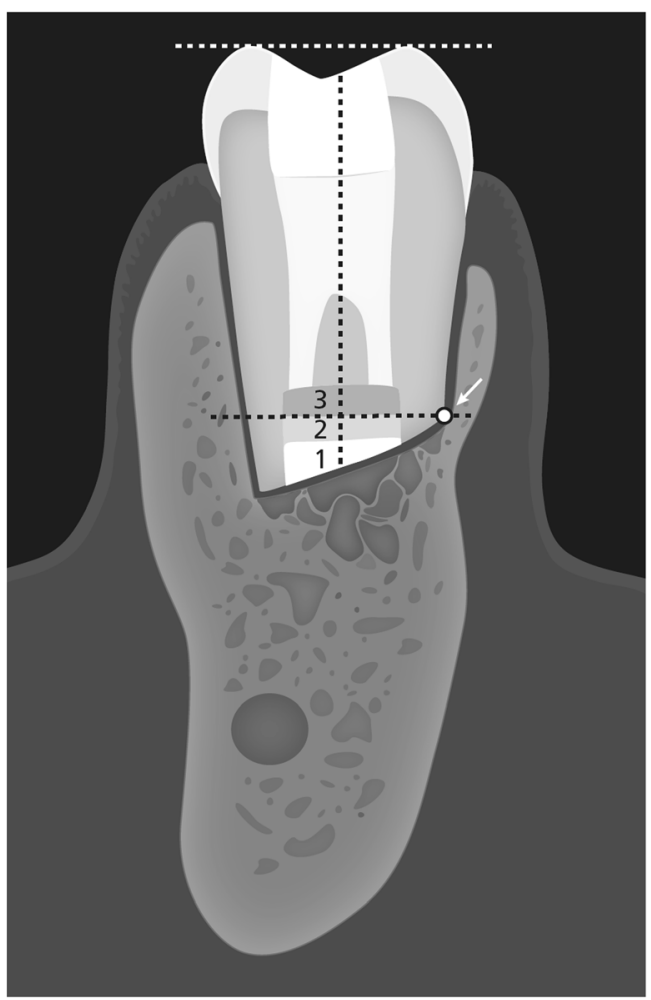

Fig. 3 Schematic illustration of the length of the root-end filling relative to a reference line through the most coronal point (white arrow) of the resection plane: length $1=$ retrofilling is inadequate (short relative to reference line); length 2 = retrofilling is flush with reference line; length $3=$ retrofilling is adequate (long relative to reference line)

its relationship with the reference line as short, flush or long.

Treated teeth were categorized into four groups: maxillary anterior teeth, maxillary posterior teeth, mandibular anterior teeth and mandibular posterior teeth. Anterior teeth included incisors and canines whereas posterior teeth comprised premolars and molars. The study parameters were correlated with the specific tooth groups.

Three independent observers rated the bucco-lingual CBCT scans with regard to periapical healing using a classification according to the radiographic criteria presented by Rud et al. [10] and Molven et al. [11]. Based on these data, overall healing was classified either as "healed" or "not healed":

- Healed: The absence of clinical signs and symptoms and complete or incomplete (scar tissue) radiographic healing.

- Not healed: The presence of clinical signs or symptoms or uncertain or unsatisfactory radiographic healing

Clinical signs and symptoms included pain; sensitivity to percussion or palpation and the presence of a fistula, a swelling or a communicating apico-marginal lesion. 


\section{Statistical analysis}

All data were first analysed descriptively. For the intraobserver reliability, Cohen's kappa values for two ratings with squared weights were calculated-meaning that weighted (Cohen's) kappa was applied. To detect significant differences in the resection angles (means), as well as in the surgical depths (means) for the different tooth groups, Kruskal-Wallis rank sum tests for the overall comparison and exact Wilcoxon rank sum tests for group-wise comparison were performed. To test any linear dependence between the angles (means) and the surgical depths (means), an $F$ test was used. To detect significant differences in the retrofilling level for the different tooth groups, Pearson's chi-square tests with simulated $p$ values were performed. The ANOVA $F$ test was considered to test for dependence between retrofilling level and resection angles (means). The influence of the resection angle (shallow $\leq 20^{\circ}$ versus acute $>20^{\circ}$ ) on treatment outcome was evaluated using the Fisher's exact test. The influence of the surgical depth $(\mathrm{mm})$ on treatment outcome was evaluated with a $t$ test. A $p$ value of $<0.05$ was chosen as the level of significance. All tests were performed using the Internet-based $\mathrm{R}$ software package (R 3.0.3; www.r-project.org).

\section{Results}

In the 48 assessed patients, a total of 62 roots in 55 teeth could be evaluated with regard to the study parameters (Table 1). Patients comprised of 22 males and 26 females with a mean age of 54.6 years (range 24-73 years). Reasons for dropouts from the initial cohort of 58 patients were as follows: patient did not attend the follow-up examination $(n=4)$, patient denied to have a CBCT taken at the follow-up $(n=4)$ or the

Table 1 Overview of evaluated teeth and roots

\begin{tabular}{llll}
\hline & & $N$ teeth & $N$ roots \\
\hline Maxilla & Central incisors & 12 & 12 \\
& Lateral incisors & 8 & 8 \\
& 1st premolars & 4 & 4 \\
& 2nd premolars & 7 & 7 \\
& 1st molars & 4 & $5(\times 4$ mesio-buccal, $\times 1$ \\
& & & disto-buccal $)$ \\
Mandible & Subtotal & 35 & 36 \\
& Canines & 2 & 2 \\
& 2nd premolars & 3 & 3 \\
& 1st molars & 15 & $21(\times 14$ mesial, $\times 7$ distal $)$ \\
& Subtotal & 20 & 26 \\
& Total & 55 & 62 \\
\hline
\end{tabular}

Table 2 Calculated resection angle $(n=62)$

\begin{tabular}{lll}
\hline & Mean $\pm \mathrm{SD}\left(^{\circ}\right)$ & Range $^{\mathrm{a}}$ \\
\hline All roots $(n=62)$ & $17.7 \pm 11.4$ & $-9.6-43.4$ \\
Anterior maxillary roots $(n=20)$ & $25.8 \pm 10.3^{*, * *}$ & $4.0-43.4$ \\
Posterior maxillary roots $(n=16)$ & $10.7 \pm 9.4^{*}$ & $-5.2-25.2$ \\
Anterior mandibular roots $(n=2)$ & $22.2 \pm 8.0$ & $16.5-27.8$ \\
Posterior mandibular roots $(n=24)$ & $15.1 \pm 9.8^{* *}$ & $-9.6-30.3$ \\
\hline
\end{tabular}

${ }^{\mathrm{a}}$ Negative values $=$ resection plane inclined towards lingual $/$ palatal aspect; positive values $=$ resection plane inclined towards labial $/$ buccal aspect

${ }^{*}$ Significant difference $(p<0.001)$; **significant difference $(p<0.05)$

quality of the CBCT did not allow a precise evaluation of the study parameters $(n=2)$.

With regard to the intra-observer reliability of measurements, the first and second readings of resection angles differed on average by $0.09^{\circ}$ with a Kappa value of 0.645 (substantial agreement). The first and second measurements of surgical depths varied on average by $0.07 \mathrm{~mm}$ with a Kappa value of 0.957 (almost perfect agreement).

The mean calculated angle of the resection plane of all roots was $17.7^{\circ} \pm 11.4^{\circ}$. The lowest mean angle was observed in posterior maxillary roots $\left(10.7^{\circ} \pm 9.4^{\circ}\right)$, whereas the highest mean angle was found in anterior maxillary roots $\left(25.8^{\circ} \pm 10.3^{\circ}\right)$ (Table 2). The location of the treated tooth had an overall significant effect on the measured angle $(p<0.001)$. The most significant differences were found between anterior maxillary and posterior maxillary roots $(p<0.001)$, as well as between anterior maxillary and posterior mandibular roots $(p<0.05)$. The frequency analysis of the calculated angles is shown in Fig. 4.

In roots with a shallow resection angle $\left(\leq 20^{\circ}\right)$, the rate of healed cases was higher than in roots with an acute resection angle $\left(>20^{\circ}\right)$ (Table 3$)$, but the difference was not statistically significant $(p=0.0905)$.

The mean measurement of the surgical depth of all roots was $6.3 \pm 1.2 \mathrm{~mm}$. The longest mean surgical depth was noted in posterior mandibular roots $(7.2 \pm 1.1 \mathrm{~mm})$, while the

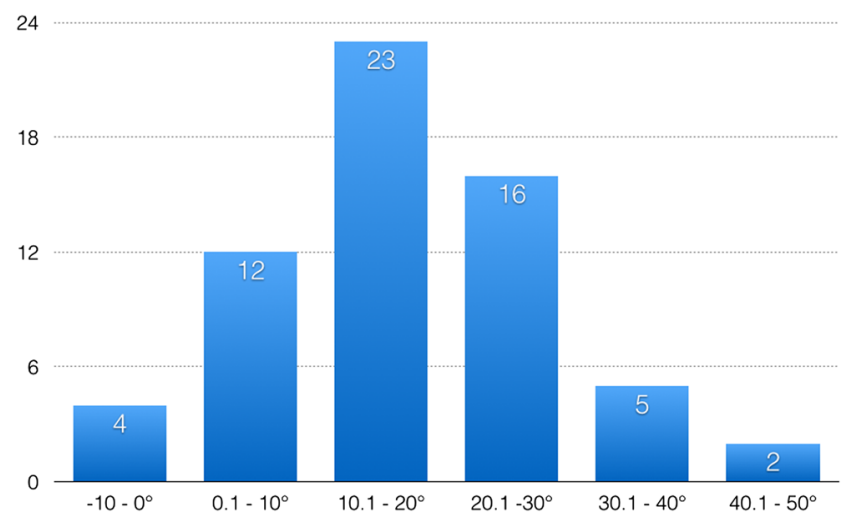

Fig. 4 Frequency of resection angles per angle categories $(n=62)$ 
Table 3 Rates of healed and not healed roots per angle categories

\begin{tabular}{|c|c|c|c|c|c|}
\hline & \multicolumn{2}{|c|}{ Healed } & \multicolumn{2}{|c|}{ Not healed } & \multirow[t]{2}{*}{ Tota } \\
\hline & $N$ & $\%$ & $N$ & $\%$ & \\
\hline Shallow angle $\leq 20^{\circ}$ & 37 & 94.9 & 2 & 5.1 & 39 \\
\hline Acute angle $>20^{\circ}$ & 18 & 78.3 & 5 & 21.7 & 23 \\
\hline Total & 55 & 100 & 7 & 100 & 62 \\
\hline
\end{tabular}

shortest mean depth was observed in anterior maxillary roots $(5.4 \pm 0.5 \mathrm{~mm})$ (Table 4$)$. The location of the treated tooth had an overall significant effect on the measured surgical depth $(p<0.001)$. More specifically, significant differences were found between anterior maxillary and posterior mandibular roots $(p<0.001)$, as well as between posterior maxillary and posterior mandibular roots $(p<0.001)$. A borderline significance was seen for anterior maxillary versus anterior mandibular roots $(p=0.052)$. There was no linear dependence between the measured surgical depth and the calculated angle of the resection plane ( $p=0.887)$.

The mean surgical depth of healed cases was $6.33 \pm 1.25 \mathrm{~mm}$ and of non-healed cases $5.62 \pm 0.70 \mathrm{~mm}$. This difference was statistically significant $(p=0.042)$.

With regard to the length of the root-end filling with regard to a reference line through the most coronal point of the resection plane, the minimal length of the retrofilling reached beyond that point in $82.3 \%$ of all evaluated cases (Table 5). However, in posterior mandibular roots, the length of the retrofilling level was significantly less frequently adequate $(p<0.05)$ compared to retrofilling lengths in the other roots. In general, cases with higher angles tended to have shorter retrofillings. However, there was no correlation between the resection angle and the length of the retrofilling $(p=0.156)$.

\section{Discussion}

The present CBCT-based study evaluated the resection angle of root apices following apical surgery. From a theoretical point of view, clinicians should bevel the root-end resection as perpendicularly to the long axis of the root as possible to

Table 4 Calculated surgical depth at resection level $(n=62)$

\begin{tabular}{lll}
\hline & Mean \pm SD $(\mathrm{mm})$ & Range $(\mathrm{mm})$ \\
\hline All roots $(n=62)$ & $6.25 \pm 1.2$ & $3.9-9.4$ \\
Anterior maxillary roots $(n=20)$ & $5.4 \pm 0.5^{\mathrm{a}}$ & $4.5-6.8$ \\
Posterior maxillary roots $(n=16)$ & $5.8 \pm 1.1^{\mathrm{b}}$ & $3.9-7.6$ \\
Anterior mandibular roots $(n=2)$ & $6.1 \pm 0.3$ & $5.9-6.3$ \\
Posterior mandibular roots $(n=24)$ & $7.2 \pm 1.1^{\mathrm{a}, \mathrm{b}}$ & $5.4-9.4$ \\
\hline
\end{tabular}

Same superscripts denote significant differences $(p<0.001)$ benefit from optimized elimination of apical ramifications combined with limited removal of root length, to reduce the area of the resection plane and amount of open dentinal tubules, and to minimize the periphery of the retrofilling at risk for bacterial leakage. However, to the knowledge of the authors, no clinical study has ever assessed the resection angle in apical surgery in humans, nor correlated the resection angle to the success rate.

Various definitions have been used in the literature to describe the optimal bevelling of root-end resection:

- Resection plane perpendicular to the long axis of the root [12]

- Resection plane without bevel [13]

- Resection plane with a $90^{\circ}$ angle relative to the long axis of the root [14-16]

- Resection plane with a $0^{\circ}$ angle relative to a line perpendicular to the long axis of the root [4]

In the present study, the latter definition was used, i.e. the resection angle was calculated relative to a reference line that was perpendicular to the long axis of the root. The calculated mean resection angle was $17.7^{\circ}$. A shallow resection angle may conserve (cortical) bone and root length. In contrast, acute bevelling of the resection plane has several risks, such as missing the lingual parts of the apex (in particular, in mandibular molars) (Fig. 5), elongating the outline of the root canal and reducing the root diameter of the non-resected part of the root apex [1]. It was interesting to note in the present analysis that in the maxilla as well as in the mandible, contrary to expectation, mean resection angles were higher in anterior compared to posterior teeth. This might be explained by the angulation of the roots, by their position within the alveolar process and by different perspectives of the surgeon in the anterior versus posterior jaw segments, respectively. One may also speculate that the surgeon would increase the bevel in roots with a long surgical depth; however, such a correlation could not be shown in the present

Table 5 Shortest length of root-end filling relative to most coronal point of resection plane $(n=62)$

\begin{tabular}{llll}
\hline & $\begin{array}{l}\text { Short } \\
\text { (apical) }\end{array}$ & $\begin{array}{l}\text { Flush (same } \\
\text { level })\end{array}$ & $\begin{array}{l}\text { Long } \\
\text { (coronal) }\end{array}$ \\
\hline $\begin{array}{l}\text { All roots }(n=62) \\
\text { Anterior maxillary roots } \\
(n=20)\end{array}$ & $8(12.9 \%)$ & $3(4.8 \%)$ & $51(82.3 \%)$ \\
$\begin{array}{c}\text { Posterior maxillary roots } \\
(n=16)\end{array}$ & $1(6.2 \%)$ & 0 & $20(100 \%)$ \\
$\begin{array}{c}\text { Anterior mandibular roots } \\
(n=2)\end{array}$ & 0 & 0 & $15(93.8 \%)$ \\
$\begin{array}{c}\text { Posterior mandibular roots } \\
(n=24)\end{array}$ & $7(29.2 \%)$ & $3(12.5 \%)$ & $14(58.3 \%)$ \\
\hline
\end{tabular}


analysis. Since an endoscope was routinely used during apical surgery, additional bevelling and/or bone removal could be avoided, thus explaining the relatively shallow mean resection angle in posterior teeth.

The effect of the resection angle on healing outcome has not been evaluated in detail in clinical studies. In a retrospective study [17] of prognostic variables, 116 teeth treated with apical surgery had a "minimal" bevel of $0^{\circ}-$ $10^{\circ}$, whereas 55 teeth had a "pronounced" bevel $>10^{\circ}$. The rate of failures was $10.3 \%$ in teeth with a minimal bevel, but $29.1 \%$ in teeth with a pronounced bevel. In the univariate analysis, this difference was significant $(p=0.003)$, but became insignificant $(p=0.061)$ after multivariate regression analysis. However, the data and results of that study must be interpreted with caution, since it was a retrospective analysis with follow-up periods ranging from 1 to 16 years. Further, the authors did not mention how they determined the bevel angle. In the present study, the rate of healed cases differed by more than $16 \%$ when comparing teeth with a shallow resection angle $(94.9 \%)$ and teeth with an acute angle $(78.3 \%)$. However, probably due to the limited number of teeth per group, this difference did not reach statistical significance.

Some experimental studies have evaluated the influence of varying the resection angle on leakage using extracted human teeth and in vitro microleakage models [4, 14-16]. While some

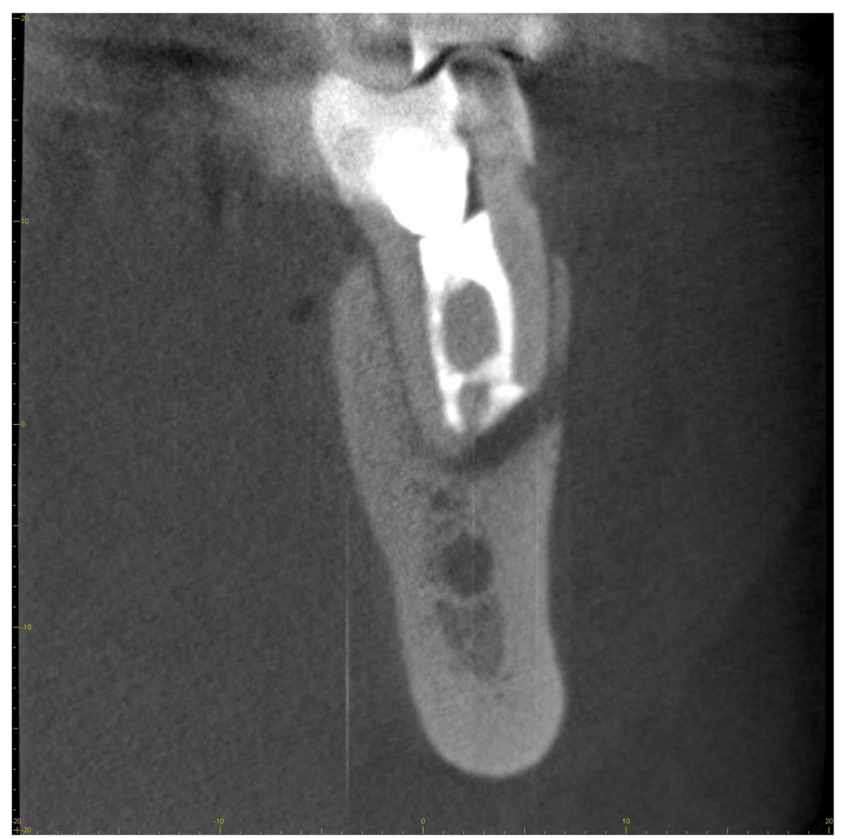

Fig. 5 A 49-year-old male patient was referred for diagnosis of persistent periapical pathosis after apical surgery of the mesial root in a first mandibular molar (case not from study sample). The bucco-lingual CBCT scan shows acute bevelling (angle of $42^{\circ}$ ) completely missing the lingual part of the root apex. The root-end filling is too shallow and seals only the mesio-buccal canal. Neither the mesio-lingual canal nor the isthmus has been retrofilled

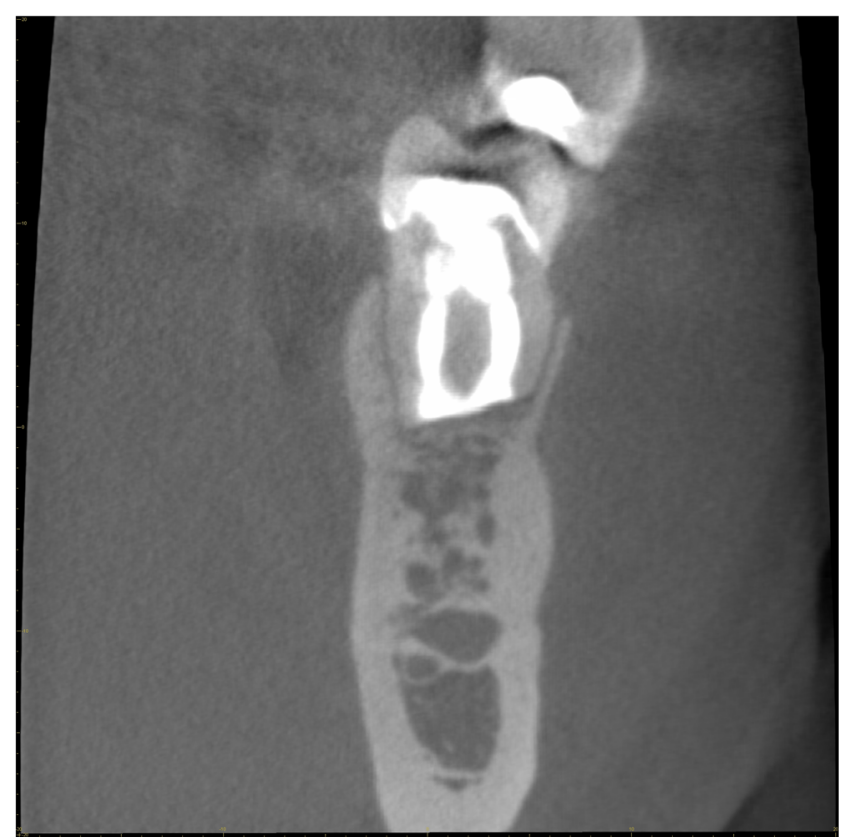

Fig. 6 Bucco-lingual CBCT scan 1 year after apical surgery of the mesial root of mandibular first molar in a 46-year-old female (case from study sample). The calculated resection angle was $12.5^{\circ}$. Both buccal and lingual canals show adequate length of the root-end filling, but in the isthmus area, the retrofilling is too short

of the studies documented a positive correlation of the resection angle and the amount of leakage [4, 14], other studies reported no association between the angle and leakage $[15,16]$. Interestingly, the latter two studies had used mineral trioxide aggregate (MTA) for root-end filling, whereas the studies showing a correlation between the angle and leakage had used glass ionomer cement [4] and zinc oxide eugenol with benzoic acid [14], respectively. The properties of MTA might have counteracted the limitations of acute bevelling. In fact, recent meta-analyses have reported MTA to be superior to other root-end filling materials in clinical outcome studies [18, 19].

The surgical depth (as defined in this study) has not been evaluated in previous studies about apical surgery. In contrast, two studies analysed a possible correlation of the depth of the bony crypt and the healing outcome 1 year after apical surgery $[20,21]$. In one study, a significant difference was reported with healed cases presenting a mean depth of $7.15 \pm 0.18$ versus $8.10 \pm 0.33 \mathrm{~mm}$ in non-healed cases $(p=0.014)$ [20]. In the second study, the depth of the bony crypt was dichotomized $(\leq 5$ versus $>5 \mathrm{~mm}$ ) [21]. Success rates were 87.5 and $88.2 \%$, respectively $(p=1.00)$. In the present study, the mean surgical depth of healed cases was $6.33 \pm 1.25 \mathrm{~mm}$ and of nonhealed cases $5.62 \pm 0.70 \mathrm{~mm}$ resulting in a borderline significance $(p=0.042)$. As mentioned earlier, a longer surgical depth did not necessarily increase the bevel of the resection plane. But the surgical depth was correlated with the type of treated roots with generally longer distances in posterior compared to anterior teeth per jaw and also longer distances in 
mandibular compared to maxillary roots. Both latter findings may be explained with the anatomical position of the roots within the alveolar process as well as with the bucco-lingual dimension of the roots.

It has been further demonstrated that the length of the root-end filling is an important issue with regard to bevelling of root apices. Gilheany et al. [4] showed that resection angles of $0^{\circ}, 30^{\circ}$ or $45^{\circ}$ required a minimum length of 1.0, 2.1 and $2.5 \mathrm{~mm}$, respectively, for preventing leakage. In general, increasing the length of the retrograde filling decreased the amount of leakage. Consequently, those authors suggested that the root-end filling should be ideally $3.5 \mathrm{~mm}$ deep since it is clinically difficult to evaluate the resection angle. One may postulate that the root-end filling should at least reach the level of the most coronal aspect of the bevelled root-end face to seal possible pathways through the resected apical dentine (patent tubules). In fact, studies have documented leakage not only along the dentine walls of retrograde fillings but also within the apical dentine [4, 14]. In the vast majority (in $82.3 \%$ ) of treated cases in the present study, the root-end filling surpassed the most coronal point of the resection bevel. However, in $41.7 \%$ of posterior mandibular roots $(n=10)$, the retrograde filling did not meet this requirement. All those cases involved first mandibular molar roots, three distal and seven mesial roots. In two of the three distal roots, long metallic posts did not allow a sufficiently deep retrofilling, whereas in all mesial roots, the retrofilling was too short in the isthmus area (Fig. 6).

\section{Conclusions}

The present study appears to be the first to have assessed and measured the resection angle in apical surgery in humans. The data allow the following conclusions:

- The majority $(62.9 \%)$ of treated roots had a shallow resection angle $\leq 20^{\circ}$.

- Cases with a shallow resection angle presented a higher healed rate than cases with an acute resection angle, but the difference was not statistically significant.

- No linear dependence of resection angle and surgical depth was observed.

- Particularly in mandibular molars, the length of the rootend filling was considered short in $29.2 \%$, especially in the isthmus area of mesial roots.

Acknowledgments The authors thank Janine Kuratli, Institute of Mathematical Statistics and Actuarial Science, University of Bern, Switzerland, for her assistance regarding the statistical analysis and Bernadette
Rawyler, Medical Illustrator, School of Dental Medicine, University of Bern, Switzerland, for the schematic illustrations.

Compliance with ethical standards All procedures performed were in accordance with the ethical standards of the institutional research committee and with the 1964 Helsinki Declaration and its later amendments or comparable ethical standards.

Conflict of interest The authors declare that they have no competing interests.

\section{References}

1. Kim S, Kratchman S (2006) Modern endodontic surgery concepts and practice: a review. J Endod 32:601-623

2. Mjör IA, Smith MR, Ferrari M, Mannocci F (2001) The structure of dentine in the apical region of human teeth. Int Endod J 346-353

3. Tidmarsh BG, Arrowsmith MG (1989) Dentinal tubules at the root ends of apicected teeth: a scanning electron microscopic study. Int Endod J 22:184-189

4. Gilheany PA, Figdor D, Tyas MJ (1994) Apical dentin permeability and microleakage associated with root end resection and retrograde filling. J Endod 20:22-26

5. Christiansen R, Kirkewang LL, Gotfredsen E, Wenzel A (2009) Periapical radiography and cone beam computed tomography for assessment of the periapical bone defect 1 week and 12 months after root-end resection. Dentomaxillofac Radiol 38:531-536

6. Tanomaru-Filho M, Jorge EG, Guerreiro-Tanomaru JM, Reis JM, Spin-Neto R, Goncalves M (2015) Two- and tridimensional analysis of periapical repair after endodontic surgery. Clin Oral Invest 19: $17-25$

7. Bornstein MM, Bingisser AC, Reichart PA, Sendi P, Bosshardt DD, von Arx T (2015) Comparison between radiographic (2D and 3D) and histologic findings of periapical lesions treated with apical surgery. J Endod 41:804-811

8. von Arx T, Hänni S, Jensen SS (2010) Clinical results of two different methods of root-end preparation and filling in apical surgery: mineral trioxide aggregate and adhesive resin composite. J Endod 36:1122-1129

9. Bornstein MM, Scarfe WC, Vaughn VM, Jacobs R (2014) Cone beam computed tomography in implant dentistry: a systematic review focusing on guidelines, indications, and radiation dose risks. Int J Oral Maxillofac Implants 29(Supplement):55-77

10. Rud J, Andreasen JO, Jensen JE (1972) Radiographic criteria for the assessment of healing after endodontic surgery. Int J Oral Surg $1: 195-214$

11. Molven O, Halse A, Grung B (1987) Observer strategy and the radiographic classification of healing after endodontic surgery. Int J Oral Maxillofac Surg 16:432-439

12. Morgan LA, Marshall JG (1998) The topography of root ends resected with fissure burs and refined with two types of finishing burs. Oral Surg Oral Med Oral Pathol Oral Radiol Endod 85:585591

13. Kontakiotis EG, Lagoudakos TA, Georgopoulou MK (2004) The influence of root-end resection and root-end cavity preparation on microleakage of root filled teeth in vitro. Int Endod J 37:403-407

14. Gagliani M, Taschieri S, Molinari R (1998) Ultrasonic root-end preparation: influence of cutting angle on the apical seal. J Endod 24:726-730

15. Post LK, Lima FG, Xavier CB, Demarco FF, Gerhardt-Oliveira M (2010) Sealing ability of MTA and amalgam in different root-end 
preparations and resection bevel angles: an in vitro evaluation using marginal dye leakage. Braz Dent J 21:416-419

16. Garip H, Garip Y, Orucoglu H, Hatipoglu S (2011) Effect of the angle of apical resection on apical leakage, measured with a computerized fluid filtration device. Oral Surg Oral Med Oral Pathol Oral Radiol Endod 111:e50-e55

17. Villa-Machado PA, Botero-Ramirez X, Tobon-Arroyave SI (2013) Retrospective follow-up assessment of prognostic variables associated with the outcome of periradicular surgery. Int Endod J 46: 1063-1076

18. von Arx T, Penarrocha M, Jensen SS (2010) Prognostic factors in apical surgery with root-end filling: a meta-analysis. J Endod 36: 957-973
19. Tsesis I, Rosen E, Taschieri S, Telishevsky Strauss Y, Ceresoli V, del Fabbro M (2013) Outcomes of surgical endodontic treatment performed by a modern technique: an updated meta-analysis of the literature. J Endod 39:332-339

20. von Arx T, Hänni S, Jensen SS (2007) Correlation of bone defect dimensions with healing outcome one year after apical surgery. J Endod 33:1044-1048

21. Song M, Gyoon Kim S, Shin SJ, Kim HC, Kim E (2013) The influence of bone tissue deficiency on the outcome of endodontic microsurgery: a prospective study. J Endod 39:1341-1345 